\title{
ÖGUM-Förderpreisträger 2013 Dr. Christian Siedentopf
}

Der diesjährige Förderpreis ist auf der Generalversammlung im Rahmen des DLT 2013 in Stuttgart Herrn Dr. Christian Siedentopf von der MUW Innsbruck mit seiner eingereichten Studie über Meralgia paraesthetica: Ultraschall gezielte Infiltration mit Follow-up-Daten über 12 Monate zuerkannt worden. Herr Dr. Siedentopf erhielt seine Ausbildung an der Otto von Guericke Universität Magdeburg und der Medizinischen Universität Innsbruck und schloß sein Medizinstudium 2002 mit der Dissertation „Das funktionelle MRI zur Untersuchung cerebraler Reaktionen auf die Stimulation von Akupunkturpunkten mit dem Akupunkturlaser“ an der Universitätsklinik für Radiologie in Innsbruck ab. Sein derzeitiger Schwerpunkt in der Facharztausbildung für Radiologie liegt beim muskuloskelettalen Ultraschall peripherer Nerven.

Im weiteren finden Sie einen Auszug aus seiner Studienbeschreibung (den Volltext finden Sie auf der ÖGUM-Website unter Förderpreisträger). Dem Preisträger noch einmal herzlichen Glückwunsch und weiterhin viele „Ultraschall-Erfolge“ !

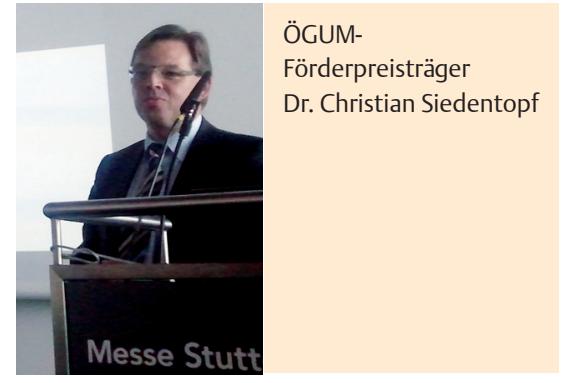

Für Patienten, die auf orale Medikamente oder konservative Maßnahmen nicht ansprechen, wird die regionale Nervenblockade der LFCN als wirksame Behandlung von Meralgia paraesthetica empfohlen. Durch eine breite Variabilität im anatomischen Verlauf des LFCN ist die Wirksamkeit der anästhetischen Blockade bei „blinder“ Applikation aber mit einer Ausfallrate von bis zu 60\% [1] eingeschränkt.

Das Ziel der vorliegenden Studie ist es, das Potenzial der Ultraschall (US) geführten Injektion von Steroiden zur Behandlung von Patienten mit Meralgia parestetica unter erstmaliger Berücksichtigung des Langzeit-Therapieerfolges über einen Zeitraum von 12 Monaten zu untersuchen.

Im Rahmen eines Pilotprojekts wurden bislang $\mathrm{n}=10$ Patienten mit den typischen Symptomen einer Meralgia paraesthetica untersucht (6 Männer, Altersdurchschnitt =63,0; 4 Frauen, Altersdurchschnitt = $65,75)$.

\section{Studienabstract} $\nabla$

Dr. Christian Siedentopf (MedUni Innsbruck) : Meralgia paraesthetica: Ultraschall gezielte Infiltration mit Follow-upDaten über 12 Monate

Co-Autoren: C. Martinoli, G. Feuchtner, M. Sojer, W. Jaschke, A. Klauser

Meralgia paraesthetica (MP) ist eine schmerzhafte Mononeuropathie des N. cutaneus femoris lateralis (LFCN), welche durch Schmerzen und / oder sensorische Störungen im Versorgungsterritorium des Nerven charakterisiert ist. 
Bei allen 10 Patienten wurde bei der Erstuntersuchung eine Schwellung des LFCN auf Höhe der ASIS (Level 1) gefunden und die Patienten wurden hier infiltriert. Der Mean-VAS betrug 85. Nach 6 Wochen waren 3 von 10 Patienten beschwerdefrei und blieben dies über 12 Monate (VAS 0). Ein Patient mussten bei persistierender Schwellung des LFCN auf Level 1 reinfiltriert werden und war bis zur Follow-UpUntersuchung nach 12 Monate beschwerdefrei.

Die restlichen 6 Patienten zeigten weitere Schwellung des LFCN unterhalb Level 1, bei 2 Patienten lag die Schwellung unterhalb des Leistenbandes (Level 2) und bei 4 Patienten am unteren Oberschenkel (Level 3). Eine völlige Beschwerdefreiheit über 12 Monate konnte bei einem von zwei Patienten auf Level 2 und bei 2 von 4 Patienten auf Level 3 erreicht werden. Die 3 Patienten mit persistierenden Beschwerden zeigten aber Beschwerdebes- serung und eine Reduktion des VSA von durchschnittlich 40 Punkten.

Insgesamt konnte eine komplette Beschwerdefreiheit in 7 von 10 Patienten über 12 Monate erreicht werden. Der VAS zeigte dabei eine Veränderung von 75,71 auf 0 ( $p<0.05$ ). Die verbliebenen 3 Patienten mit persistierenden Beschwerden zeigte aber eine deutliche Reduktion der Beschwerden über 12 Monate (VAS von 86,6 auf 46). Es gab keine untersuchungsoder therapiebedingten Komplikationen.

Diese Studie bietet zum ersten Mal in der Literatur Follow-up-Daten zu US-geleiteten Injektionen bei MP über einen Zeitraum von 12 Monaten. Die nachgewiesene und anhaltende vollständige Beschwerderückbildung bei 7 der 10 Patienten und die deutliche Reduktion der Beschwerden bei 3 der 10 Patienten über 12 Monate unterstreicht die Bedeutung der Verwendung des Ultraschalls für die visu- ell kontrollierte Infiltration des LFCN. Neben der exakten anatomischen Lage können auch gezielt die geschwollenen Anteile des LFCN aufgesucht und infiltriert werden. Auf der Grundlage dieser Daten empfiehlt sich die genaue Ultraschall-gestützte Untersuchung des gesamten Nervenverlaufs und die gezielte Infiltration der geschwollenen Abschnitte auch unterhalb des Level 1. Durch die punktgenaue Infiltration kann außerdem die Menge an Triamcinolon je Infiltration deutlich reduziert werden, sodass nur $1 / 4$ der üblichen $40 \mathrm{mg} / \mathrm{ml}$ verabreicht wurden.

Diese ersten Resultate der Pilotstudien bilden die Basis für eine auf 2 Jahre angelegte Follow-up-Untersuchung in der die genannten Verbesserungen methodisch implementiert werden und welche aufgrund einer größeren Anzahl von Untersuchungen die Resultate statistisch besser absichern kann. 